\title{
EXTRACTIVISMO CORPORATIVISTA EN MÉXICO: MINERÍA EN GUANAJUATO
}

\author{
CORPORATIVIST EXTRACTIVISM IN MEXICO: MINING IN GUANAJUATO
}

EXTRATIVISMO CORPORATIVISTA NO MÉXICO: MINERAÇÃO EM GUANAJUATO

\section{Aleida Azamar Alonso ${ }^{1}$}

\begin{abstract}
Resumen
En este trabajo se realiza un estudio de la minería metálica en México, en el estado de Guanajuato, donde las condiciones de mercado han creado un escenario de monopolización por solo dos compañías canadienses. Esta conducta responde a la transición económica del capitalismo hacia su fase superior, en la que los medios de producción y el poder político se concentran en una mínima cantidad de actores. El análisis se realizó a través del diagnóstico productivo minero del Estado; asimismo, se valoran elementos teóricos marxistas que ayudan a determinar el proceso de corporativismo y la monopolización extractivista.
\end{abstract}

Palabras clave: minería; extractivismo; monopolio

\begin{abstract}
In this paper, a study of metallic mining is conducted in Mexico, in the state of Guanajuato, where market conditions have created a scenario of monopolization by only two Canadian companies. This behavior responds to the economic transition of capitalism to its higher phase, in which the means of production and political power are concentrated in a minimum number of actors. The analysis was carried out through the productive mining diagnosis of the State; likewise, Marxist theoretical elements that
\end{abstract}

Doi: https://doi.org/10.15359/eys.24-55.5

Fecha de recepción: 16-01-2019. Fechas de reenvíos: 05-03-2019, 13-03-2019, 20-03-2019. Aceptado el 26-03-2019. Publicado el 28-03-2019.

${ }^{1}$ Profesora e investigadora del Área de Análisis y Gestión Socioeconómica de las Organizaciones. Departamento de Producción Económica, Universidad Autónoma Metropolitana, Unidad Xochimilco, Ciudad de México, México. Correo electrónico: gioconda15@gmail.com https://orcid.org/0000-0002-7915-7611

78

Aleida Azamar Alonso

(c) (i) 8 ()

Revista Economía y Sociedad by Universidad Nacional is licensed under a CreativeCommons Reconocimiento-NoComercial- 
help determine the process of corporativism and extractivist monopolization are valued.

Keywords: mining; extractivism; monopoly

\begin{abstract}
Resumo
Neste trabalho é realizado um estudo da mineração metálica no México, no estado de Guanajuato, onde as condições de mercado criaram um cenário de monopolização por apenas duas companhias canadenses. Este comportamento responde à transição econômica do capitalismo para sua fase superior, na qual os meios de produção e o poder político estão concentrados em um número mínimo de atores. A análise foi realizada através do diagnóstico produtivo mineiro do Estado. Da mesma forma, são avaliados elementos teóricos marxistas que ajudam a determinar o processo de corporativismo e a monopolização extrativista.
\end{abstract}

Palavras-chaves: mineração; extrativismo; monopólio

\title{
Introducción
}

En la actualidad la mayor parte de los bienes consumidos por los seres humanos son obtenidos a través de la explotación intensiva de la naturaleza, los cuales son comerciados para su utilización industrial, por lo que se ha cambiado de acumulación hacia intercambio masivo. De forma adicional, aunque en menor medida, también sirven como reserva monetaria a nivel internacional. De esta manera, la extracción intensiva de recursos minerales ("extractivismo") se ha convertido en la columna vertebral del modelo productivo capitalista que está comenzando a trascender hacia una etapa que puede ser denominada monopolista, ya que la mayor parte del universo empresarial será desplazado por megacorporaciones que dominarán el mercado y que están siendo respaldadas por el Estado, el cual asumirá el papel de guardia de las condiciones productivas.

Este trabajo se fundamenta en los argumentos marxistas sobre las fases del sistema económico para realizar un análisis teórico del capitalismo monopolista y su influencia de la minería en Guanajuato, México. Se analiza el tema en cuatro apartados. En el primero se realiza la descripción metodológica y se mencionan los materiales empleados. En la segunda parte se establece el criterio conceptual que se emplea para los términos: extractivismo y extractivismo corporativista. En la tercera sección se perfilan los principios teóricos marxistas que ayudan a definir el proceso de transición en el capitalismo moderno, el cual conduce hacia la etapa de monopolización productiva. En el cuarto apartado se aborda la condición minera de Guanajuato 
y las empresas que se encuentran activas; asimismo, se hace una breve referencia sobre estas para después centrarse solo en una y analizar los impactos e influencia que tiene en el nivel regional, así como los elementos que servirán para determinar si existe extractivismo corporativista en esta entidad. Por último, se presentan las conclusiones.

\section{Metodología}

Para el desarrollo del concepto de extractivismo corporativista y la influencia negativa de estas actividades productivas en el desarrollo social en México, se utilizó la metodología cualitativa y cuantitativa. Lo anterior con la finalidad de abordar dos ejes en este trabajo: el primero, el análisis teórico del capitalismo desde la óptica marxista; el segundo, la valoración productiva y económica de las dos únicas empresas extranjeras en Guanajuato que actualmente mantienen proyectos activos de minería metálica en el lugar.

Para la metodología cualitativa se parte de la perspectiva marxista clásica sobre las etapas en las que transita el capitalismo para alcanzar un estado de realización completo, que se vincula primero con el proceso de privatización de la naturaleza $y$, en una segunda etapa con el fortalecimiento de los monopolios de explotación intensiva ambiental. En la revisión del tema se consideraron las aportaciones de la economía clásica y neoclásica sobre la necesidad de que exista un universo empresarial basto y libre para que funcione correctamente la economía, las cuales se refutan para el sector minero del estado de Guanajuato, a través de la demostración de una concentración territorial y productiva mediante la revisión y contrastación con datos del Servicio Geológico Mexicano (SGM) en cuanto al número de proyectos mineros activos, la distribución de los mismos y la posición dominante de pocas empresas sobre el mercado nacional.

En lo que respecta a la metodología cuantitativa se consideraron las estadísticas productivas y económicas de dos de las minas extranjeras canadienses que se ubican en el lugar. Los datos se obtuvieron del SGM (2017), así como del informe anual económico de las minas en cuestión (Endeavour Silver Corporation, 2017). De esta forma, se consideró el volumen de producción de onzas de plata y onzas de oro durante el periodo 2007 a 2016. Igualmente, se valoró la extensión y cercanía de estos proyectos al municipio de Guanajuato (SGM, 2017), una de las áreas urbanas más pobladas del estado de Guanajuato.

Este trabajo se centra en dos unidades mineras: Bolañitos y El Cubo, las cuales pertenecen a la compañía canadiense Endeavour Silver Corporation (ESC). La importancia de enfocarse en estos

80

Aleida Azamar Alonso

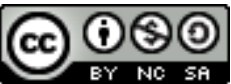

Revista Economía y Sociedad by Universidad Nacional is licensed under a CreativeCommons Reconocimiento-NoComercial- 
dos proyectos se debe a que estos representan $70 \%$ de la producción metálica de la entidad de Guanajuato, la cual es la más importante de la zona del Bajío² (SGM, 2017).

Para la localización espacial de las unidades mineras, así como de las concesiones en el estado de Guanajuato se optó por utilizar la herramienta gráfica independiente de la agencia SubVersiones realizada por Romeo LopCam. Dicha herramienta está basada en la información del Sistema de Administración Minero (SIAM) mexicano de la Secretaría de Economía (SE), enriquecido con información del Directorio Estadístico Nacional de Unidades Económicas (DENUE), actualizado hasta septiembre del 2018, por lo que contiene información más amplia, explícita y mejor ordenada que la herramienta del SIAM CartoMinMex, la cual carece de varias capas de información que sí son visibles en el recurso empleado.

Dado que el objetivo de este trabajo es señalar la existencia específica de un proceso de monopolización extractivista dentro del Bajío, el análisis se centra en probarlo sin establecer una contrastación en el nivel nacional o regional, ya que cada estado (entidad) del país tiene características complejas y diferenciadas, lo que probablemente no permitiría extrapolar las conclusiones de este trabajo a toda la Nación. No obstante, la aportación principal es probar que existe una participación del gobierno en la creación del corporativismo monopolista en este lugar.

\section{Extractivismo y corporativismo: conceptualización}

El concepto de extractivismo se ha empleado en la literatura ecológica y ambiental internacional desde hace varias décadas. Aunque su significado ha variado a lo largo del tiempo y se ha transformado en los estudios tradicionales sobre el medio ambiente ${ }^{3}$. El término era utilizado de forma genérica para referirse a todos aquellos procesos de aprovechamiento de la naturaleza; así por ejemplo, Borgtoft y Baslev (1993) señalan que dicha expresión "es la cosecha o extracción de productos de plantas que crecen de forma silvestre" (p. 5); mientras que Ruíz y Sayer (1993) estiman que este concepto se enmarca en "las actividades de caza o recolección para fines de comercialización y/o autoconsumo"(p. 3), al ordenar estas en las categorías de subsistencia, comercial y destructivas. Dufour (1990) por su parte, vincula esta concepción con los sistemas agrícolas indígenas de Brasil, los cuales, señala, son sostenibles, ya que hace énfasis en las dinámicas de recolección. Por otro lado, también se puede considerar la perspectiva de Fearnside (1988) quien establece que el vocablo se refiere a "la obtención de bienes forestales no maderables" (p. 3).

\footnotetext{
${ }^{2}$ El Bajío es una región del centro-norte del país formada por algunos municipios de seis entidades (esto se aborda en el apartado de minería en Guanajuato). Asimismo, dicha entidad ocupa el décimo tercer lugar en la aportación minera nacional.

${ }^{3}$ En los análisis clásicos de la geografía de los recursos naturales (Brunhes, 1920) se establece que las economías basadas en la extracción de materias primas son modelos directamente destructivos y de rapiña.
} 
Desde finales del siglo XX, dicha apreciación cambió, sobre todo como resultado del dramático incremento comercial en la demanda y producción de materias primas ${ }^{4}$, la cual se concentró de forma particular en América Latina y otras regiones en vías de desarrollo, que presionan a sus ecosistemas por la intensidad de la extracción de estos bienes, lo que además iba aparejado a las múltiples reformas estructurales que favorecían la reprimarización ${ }^{5}$ de estas naciones.

Es en este escenario que el concepto de "extractivismo" enriquece un campo de estudio enfocado en diferentes perspectivas de la explotación territorial y ambiental. Autores como Acosta, Alimonda, Azamar, Balanyá y Ortega, Composto, Giarraca y Teubal, Gómez, Gudynas, Portillo, Seoane, Svampa, entre muchos otros, crean y nutren diversas formas y perspectivas de estudiar este fenómeno. De manera común y a grandes rasgos (ya que no existe una definición única o primaria), se entiende que se trata de todas aquellas actividades antropogénicas que emplean o extraen grandes cantidades de materias primas para comerciarlas, sin ningún tipo de transformación, en el nivel internacional, sin favorecer a los mercados locales de donde se obtienen.

En este trabajo se emplea y amplía, la definición de Azamar (2017) en la que se establecen criterios multidimensionales para redefinir la conceptualización con base en el interés de estudiar el escenario mexicano y en específico, el del estado de Guanajuato. Por lo cual se considera que el extractivismo se refiere a aquellas actividades que emplean un volumen de materias primas superiores a las que son necesarias para cubrir la demanda local; que además, durante el proceso de extracción contamina de forma irreversible el área donde se lleva a cabo; debilita de manera estructural a la Nación, al fomentar el vínculo de dependencia externo, puesto que está enfocado al comercio internacional; depende de un marco normativo local que respalda el despojo territorial (entendido como la práctica de apropiación, legal o ilegal, de espacios ocupados en los que se encuentre una gran cantidad de recursos aprovechables para fines privados) y, también, resulta fundamental para evitar la caída de la tasa de beneficio ${ }^{6}$ del capital. Dicha interpretación

\footnotetext{
${ }^{4}$ Raw materials es como se les identifica en inglés y generalmente, son categorizados en cuatro grupos: metales, fósiles, no metales y biomasa, mismos que son contabilizados en su conjunto para medir los flujos de materiales en el mundo (Materialflows, 2019).

${ }^{5}$ Slipak (2013), explica el origen de la reprimarización de la siguiente forma: "Las primeras apariciones concretas de la expresión "reprimarización" de la economía, aparecen en alusión a las consecuencias que han traído las políticas de liberalización financiera que han seguido varias dictaduras de América Latina y posteriormente la aplicación de las prescripciones del Consenso de Washington. Precisando, la expresión "reprimarización de la economía", aparece describiendo las etapas de valorización financiera, en donde simultáneamente se observaba una tendencia a la reorientación de la economía hacia las actividades agropecuarias, con la consecuente destrucción del tejido industrial, pérdida de empleos y efectos regresivos en la distribución del ingreso" (p. 6).

${ }^{6}$ De acuerdo con Marx (2003), la tasa de ganancia es el cociente entre el plusvalor y la inversión (fija y variable). Azamar (2017), reinterpreta los cálculos de Marx para aplicarlos a la minería en México, al utilizar indicadores estadísticos nacionales actuales y los denomina tasa de beneficio, como referencia a la rentabilidad de las actividades extractivas.
}

82

Aleida Azamar Alonso

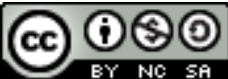

Revista Economía y Sociedad by Universidad Nacional is licensed under a CreativeCommons Reconocimiento-NoComercial- 
se ha mantenido en constante cambio y crecimiento, ya que es resultado de múltiples trabajos de investigación, por lo cual se adhiere al interés particular de este artículo.

Dado que esta apreciación no se centra en un solo tipo de proceso productivo (minero, hídrico, agrícola, pesquero, eólico, forestal, petrolero, etc.) se usa de forma indistinta la expresión "proyectos" y "actividades extractivas" para referirse a las labores productivas que se pueden categorizar dentro de la definición ya comentada. De igual forma las "empresas extractivas" son aquellas que se centran en la explotación de bienes naturales, de acuerdo con la noción mencionada.

Por otro lado, el corporativismo es por sí mismo un tema amplio y complejo, el entendimiento de este término se refiere a múltiples apreciaciones directamente relacionadas con el proceso histórico en el que se plantearon. Por ejemplo, la que se relaciona con la postura fascista de principios del siglo XX y se establece como la intervención absoluta del Estado en el desarrollo de grupos públicos o privados para la construcción de dinámicas sociales (De la Garza, 2006). Esta definición no será empleada en este trabajo porque la intención es centrarse en la propuesta clásica de Schmitter (1974) sobre "un sistema de representación de intereses en el cual, las partes constitutivas están organizadas dentro de un número de categorías singulares obligatorias [...] a las que se les concede un deliberado monopolio por representación, dentro de sus respectivas categorías [...]" (p. 93)

Al considerar lo comentado por Schmitter, el autor hace referencia a la creación de estructuras políticas de representación de grupos sociales ante el Estado, para la adquisición de derechos o beneficios que dependen de la capacidad de este para regular el escenario donde se desarrolle el corporativismo. No obstante, Lijphart $(\underline{2000})$ señala que al ser grupos de poder que no están previstos por el modelo político tradicional, se transforman en elementos que adquieren potencial de intervenir en el desarrollo de la política pública para sus propios beneficios.

Si se considera que México es un país con una larga y profunda historia ligada a la explotación ambiental, en particular a la minera (de la que además fue dependiente desde el siglo XVI hasta principios del siglo XX), es entendible como los representantes de los intereses de esta industria tuvieron importante y notable dominio en el desarrollo de la historia nacional. En este sentido, y a fin de considerarse como elemento de análisis en este trabajo, el "extractivismo corporativista" se refiere a la creación de estructuras políticas ligadas al fundamento del Estado para impulsar redes empresariales que acaparan la propiedad de recursos naturales nacionales para uso privado.

Dado que este tipo de organizaciones u ordenamientos empresariales están enfocados en fortalecer el control exclusivo del mercado a través del soporte de la administración pública se explicita a continuación el proceso que ha desarrollado esta estructura de acumulación y 
apropiación territorial en pocas manos. Por lo cual, también se expresa en el marco del capitalismo monopolista, término que se emplea para identificar este concepto.

\section{Capitalismo y monopolio}

De acuerdo con la teoría marxista, todo modelo económico-político que rige a la sociedad y se estructura sobre el conflicto de una lucha de clases tiende a pasar por dos fases: a) inferior (transición) y, b) superior (imposición). En la primera etapa se modelan y fortalecen las fuerzas productivas, se crean dinámicas de trabajo, así como reglas operativas. Por otro lado, la transición histórica a la segunda etapa conduce al conflicto con las fuerzas productivas, pues estas imponen una traba de su propio desarrollo (Marx y Engels, 1980).

Se considera que la primera fase fue la de la manufactura y, la transición hacia el ciclo industrial es la segunda, con todos los conflictos que implicó dicho tránsito. Este proceso de fabricación manual a gran escala surge en la etapa superior del feudalismo por lo que es más bien una herencia que ha de modificarse durante el periodo de cambio hacia el nuevo modelo de producción.

Las fuerzas productivas principales le dan forma a cada etapa de fabricación, trascienden hacia un nuevo modelo y entran en contradicción con la primera fase. En el caso de lo que sucede en el periodo feudal, los artesanos y campesinos son sustituidos por un trabajo masivo apoyado, parcialmente, por maquinaria tecnificada, lo que termina por desbancar tanto a la nobleza como al clero frente a los burgueses, estos últimos son quienes monopolizan los medios de producción y materializan las condiciones para orientar el capitalismo.

En este último caso, la primera fase requiere la existencia de un universo amplio de competencia con múltiples empresas que fortalecen al libre mercado y en el cual están condicionadas las reglas de oferta y demanda (Baran y Sweezy, 2006). Sin embargo, y como lo señala Marx (2003), la dominación de esta estructura socioeconómica se orienta inexorablemente hacia un escenario en el que los expropiadores de los bienes comunes (pequeños productores) también son despojados para garantizar la supervivencia del sistema, pues se articulan en una cada vez menor y más homogénea figura concentrada de grandes empresarios ${ }^{7}$.

Esta tendencia centralista del sistema económico es la etapa superior, la del monopolio en el que se financia a sí mismo y se expande por medio de un puñado de sujetos que imponen las normas

\footnotetext{
${ }^{7}$ En la actualidad el sector alimentario de Estados Unidos cuenta con más de 200 marcas y submarcas de productos o servicios, los cuales son propiedad directa o indirecta de 10 grandes consorcios, entre los que destacan: Pepsico, The Coca Cola Company, Kraft, Nestlé, P\&G, Kellogg's, Johnson \& Johnson, etc. Lo mismo sucede en las telecomunicaciones y para todos los grandes sectores productivos y de servicios.
}

84

Aleida Azamar Alonso

(c) (P) $\$$ (O)

Revista Economía y Sociedad by Universidad Nacional is licensed under a CreativeCommons Reconocimiento-NoComercialCompartirlgual 4.0 Internacional License.

Creado a partir de la obra en http://www.revistas.una.ac.cr/index.php/economia 
de producción y explotación, ya sea en el ámbito real o ficticio (Baran, 1987; Bellamy, 2014; Ceceña y Ornelas, 2016; Vladimir, 2012).

Esta fase es por la que la humanidad pasa actualmente, la concentración de las formas de reproducción del capital, en la que todas estas son respaldadas por diversas expresiones políticas y jurídicas, en donde el beneficio económico se convierte en la piedra angular para los tomadores de decisiones -mismos que comparten intereses corporativos, pues también el Estado está cooptado por el capitalismo- para asegurar su ciclo de vida. Es así como todas las relaciones productivas (trabajo, aprovechamiento de la tierra, etc.) se centran en generar ganancias para un pequeño grupo de beneficiarios (véase Figura 1).

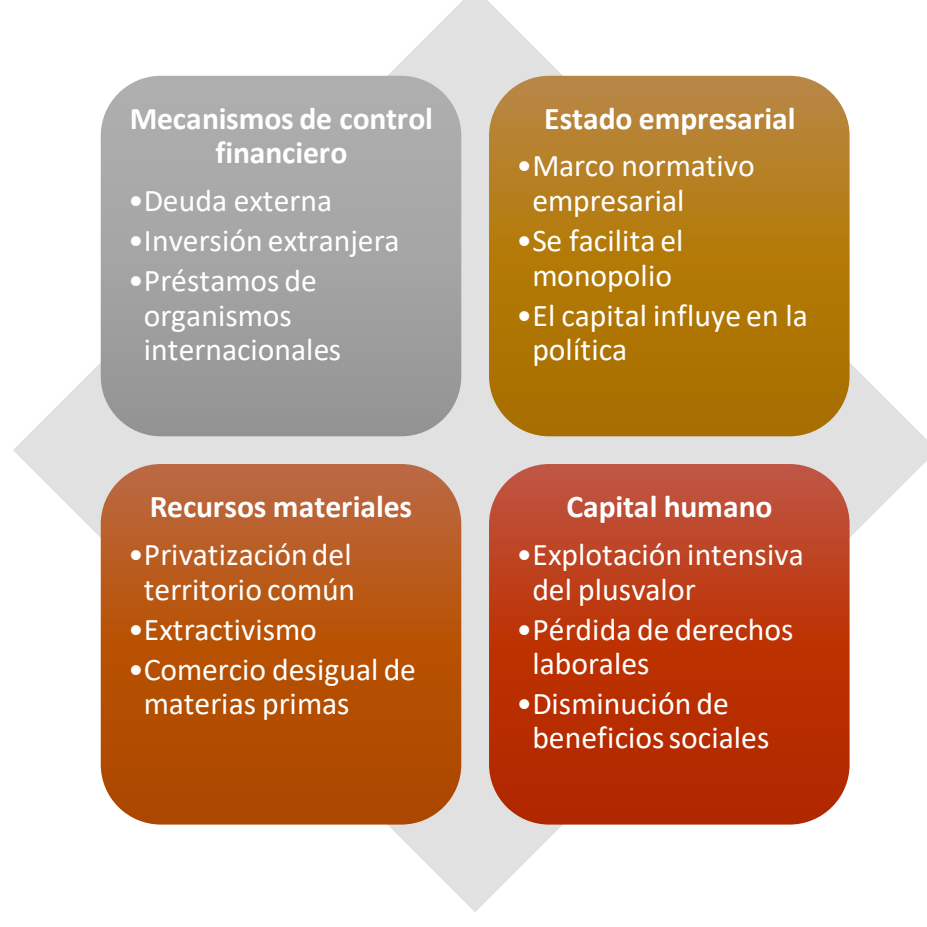

Figura 1. Caracterización del capitalismo monopolista. Fuente: elaboración propia

En la Figura 1 se intenta simplificar algunas de las principales características que identifican al régimen contemporáneo que tiene fines acaparadores y que se sirven del extractivismo para fortalecer su estructura productiva. Esta es una matriz representativa de los aspectos económicos, políticos, sociales y ambientales fundamentales para asegurar el desarrollo de este sistema productivo.

En los extremos inferiores derecho e izquierdo se observan las necesidades básicas e irremplazables de la plutocracia, los trabajadores y las materias primas. Aunque en esta parte se muestra la forma en que se garantiza su uso o se afecta su valor para beneficio privado. La parte 
de capital humano contiene los elementos relacionados con la flexibilización laboral hacia un enfoque más práctico de los mercados de trabajo en los que los derechos humanos son secundarios para los empleadores. En el segmento de recursos materiales se presenta la práctica de dominio de la naturaleza por etapas: expoliación de los medios de producción, explotación y exportación.

En la parte superior se encuentran las condiciones necesarias para ejecutar una usurpación y despojo intensivo de los bienes naturales sin preocuparse por la respuesta social o las demandas de respeto a la soberanía de las naciones. Del lado izquierdo se observan los procesos mediante los cuales las naciones desarrolladas impulsan obligaciones (deuda, intereses y carencia de capital local para invertir), para generar condiciones adversas de negociación con los países periféricos de donde se obtiene la mayor parte de los bienes naturales en la actualidad. En el extremo derecho superior el resultado es un Estado que se enfoca en beneficiar a cierto tipo de empresas y carece de voluntad para el bienestar social.

Con la finalidad de garantizar que este sistema económico pueda realizarse, es fundamental establecer un proceso iterativo que reproduzca y profundice cada uno de los elementos en el diagrama. Esto se debe a que el capitalismo necesita crear un conjunto de normas y controles sociales, políticos y económicos (obligaciones fiscales, reglas corporativistas para la población, etc.), para asegurar la existencia de un acceso privado a los bienes naturales y humanos.

$Y$ es que una de las principales particularidades de este modelo productivo es el alto consumo de materias primas para alimentar a la industria. Por ello, los espacios comunes donde se puede disfrutar de un medio ambiente saludable son privatizados e identificados como capital natural ${ }^{8}$, por lo que se vuelven una simple despensa de recursos para las empresas.

Por otra parte, la población se convierte en capital humano ${ }^{9}$ que no solamente debe ser barato sino remplazable de manera fácil, para este efecto se crean andamiajes educativos orientados a la formación técnica en masa de los trabajadores, de forma tal que se incorporen a la producción tan rápido como sea posible.

Por ello, el Estado es la entidad que garantiza a las empresas el acceso a los bienes naturales, además de que impone la normatividad para que las personas sean empleadas en condiciones ventajosas para la industria. Con la finalidad de que el Gobierno pueda ser eficiente en dicho

\footnotetext{
${ }^{8}$ El concepto de "capital natural" se refiere a las unidades atomizadas de la naturaleza que pueden ser transformadas, comerciadas y empleadas en el sistema productivo. Su uso es de carácter neoclásico (Nadal, 2015).

${ }^{9} \mathrm{Al}$ igual que en el caso anterior, es un concepto que despoja a las personas de su calidad extraordinaria para crear valor (plusvalor) y los convierte en un mero producto desechable (Nadal, 2015).
}

86

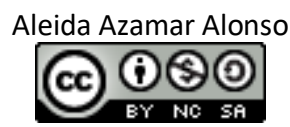

Revista Economía y Sociedad by Universidad Nacional is licensed under a CreativeCommons Reconocimiento-NoComercial- 
cometido, su estructura institucional cuenta con: organismos militares, económicos, educativos, laborales, etc., para coaccionar y asegurar sus objetivos (Ceceña, 2016).

Lo anterior responde a una disputa empresarial global sobre la hegemonía productiva y, por lo tanto, de la capacidad de acceder a los recursos naturales y a la fuerza humana, en la que los vínculos del Estado se fortalecen con las megacorporaciones para alcanzar un "desarrollo, control y gestión o monopolización de la producción estratégica y de los elementos estratégicos de la reproducción" (Ceceña y Barreda, 1995, p. 27).

Como bien cabe suponer, cada Nación-empresa competitiva está restringida a sus propias capacidades de imponer reglas de mercado, amenazas militares o de uso de fuerza y tecnología. De esta manera es que algunas regiones acatan el papel de suministradoras y otras las de transformadoras (Ceceña y Barreda, 1995).

Con el fin de sustentar esta práctica extractiva depredadora y monopolista se otorgan permisos políticos basados en dictámenes economicistas sobre el libre mercado de una supuesta democracia representativa que más bien trata de exaltar la competencia individualista antes que el bienestar común, se prioriza complacer a los mercados y actores externos, ya que es algo fundamental para la supervivencia de cada país.

De esta forma es que las naciones desarrolladas justifican la externalización de sus actividades extractivas hacia regiones donde, son necesarias para incrementar su supuesta competitividad; por otro lado, los países periféricos se vuelven laxos en sus regulaciones extractivas, con la finalidad de atraer inversión para el desarrollo de estos proyectos.

Lo anterior favorece la estructura capitalista en varios sentidos, pues los países centrales crean subsidiarias en naciones subdesarrolladas que dotan a estas empresas de un mayor capital tecnológico, lo que les permite ser más rentables al ser innovadoras, obtener ventaja competitiva y amortizar los costos de inversión, lo que afecta la competencia local, destruye las cadenas de valor internas y margina a los actores más pequeños. Estos elementos son empleados para monopolizar las actividades de explotación y aprovechamiento natural; por ejemplo, en México 9 \% de la producción nacional en 2016 correspondió a compañías con capital nacional (48 mil millones de pesos) (SGM, 2017).

Asimismo, en nuestro país la intensificación de las actividades productivas en el sector minero y de otras materias primas son una necesidad básica del capitalismo monopolista, pues este se basa en las ganancias financieras ficticias, pero depende del acceso físico a los recursos naturales para 
la generación de expectativas de producción positivas que fomenten la inversión y, por lo tanto, el crecimiento de la economía ${ }^{10}$.

El extractivismo monopolista se sitúa en la concentración de ganancias de un reducido número de empresas, ejemplo de ello es que tan solo en México existen 345 compañías que controlan más de 1100 proyectos mineros activos actualmente, lo que ocupa más del $40 \%$ del territorio nacional (Azamar, 2017; SGM, 2017). No obstante, solamente ocho son protagonistas debido a su volumen de producción, inversión y cantidad de trabajadores empleados, las cuales son: Fresnillo PLC, Goldcorp, Minera Frisco, Industrias Peñoles, Grupo México, Corporation First Majestic, Panamerican Silver Corp. y Agnico Eagle México (Rivera, 2017).

Y aunque existen varios casos sobre proyectos mineros en México, la intención en este trabajo es centrarse en la zona del Bajío, por lo que el análisis se enfoca en el estado de Guanajuato que pertenece a esta región del país, ya que se observa la creación de un clúster minero monopolista que se ha fortalecido durante el presente siglo.

\section{Corporativismo minero en Guanajuato}

Guanajuato es un estado localizado en la zona centro-norte de México y pertenece al sector conocido como El Bajío, una megalópolis o ciudad-región formada por varias áreas urbanas colindantes densamente pobladas y con una relación funcional estrecha sobre sus actividades industriales y productivas. Dicha área se integra parcialmente por las entidades de Aguascalientes, Querétaro, Jalisco, San Luis Potosí, Michoacán y Guanajuato.

Este último se divide en 46 municipios y es pequeño en comparación con el resto de las entidades nacionales, pues solamente representa $1.56 \%$ del territorio nacional (Instituto Nacional de Estadística y Geografía, 2019). No obstante, su aportación productiva a la minería es notable, pues desde la época colonial ya era considerado de gran importancia. En el año 2017 se posicionó entre los primeros diez productores de oro y plata del país (ocupa el sexto lugar a nivel nacional), siendo una de las regiones del Bajío con mayor importancia en este rubro, encontrándose por debajo de San Luis Potosí (Cámara Minera de México, 2019).

Desde el año 2005 hasta el 2017 la superficie concesionada para actividades de exploración y explotación en esta entidad ha crecido de 170 mil hectáreas (ha) a 309 mil ha, con aproximadamente 631 permisos vigentes. Lo que significa que se ha incrementado en extensión $81 \%$ en 12 años. La mayor parte de estas se encuentran en la zona centro de la entidad lo que cubre $89.9 \%$ del municipio de Guanajuato (véase Figura 2).

\footnotetext{
${ }^{10}$ Esta explicación es fundamental para el desarrollo capitalista, pues justifica el expansionismo de la apropiación territorial y las prácticas economicistas dentro de la política que beneficia a los mercados.
}

88 


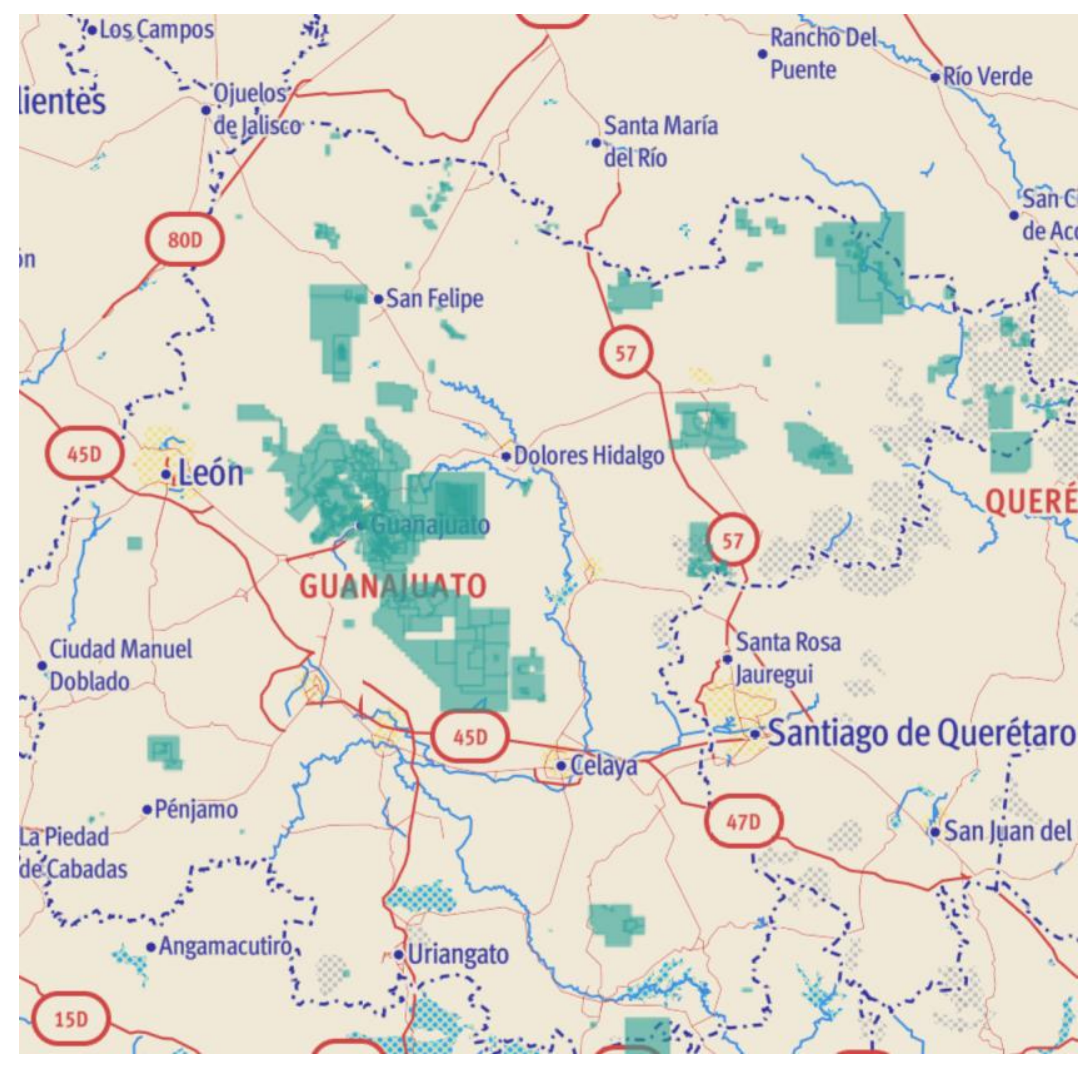

Figura 2. Mapa de concesiones en el estado de Guanajuato.

Fuente: Tomado de Romeo LopCam, 2018

Aunque la extensión de las concesiones en esta entidad es muy elevada, solamente existen tres proyectos activos dedicados a la explotación de minerales preciosos y de cobre. El restante son 48 de no metálicos, principalmente de grava y arena, que se encuentran en al menos 20 municipios del Estado (SGM, 2017).

Un detalle importante es que el nivel de producción de minerales metálicos depende, exclusivamente, de dos empresas extranjeras (ambas canadienses): 1) Endeavour Silver Corp., con dos unidades mineras y, 2) Great Panther Silver LTD, con una sola unidad (véase Figura 3). La suma de extracción diaria de estas dos compañías es de 2300 toneladas (SGM, 2017). 


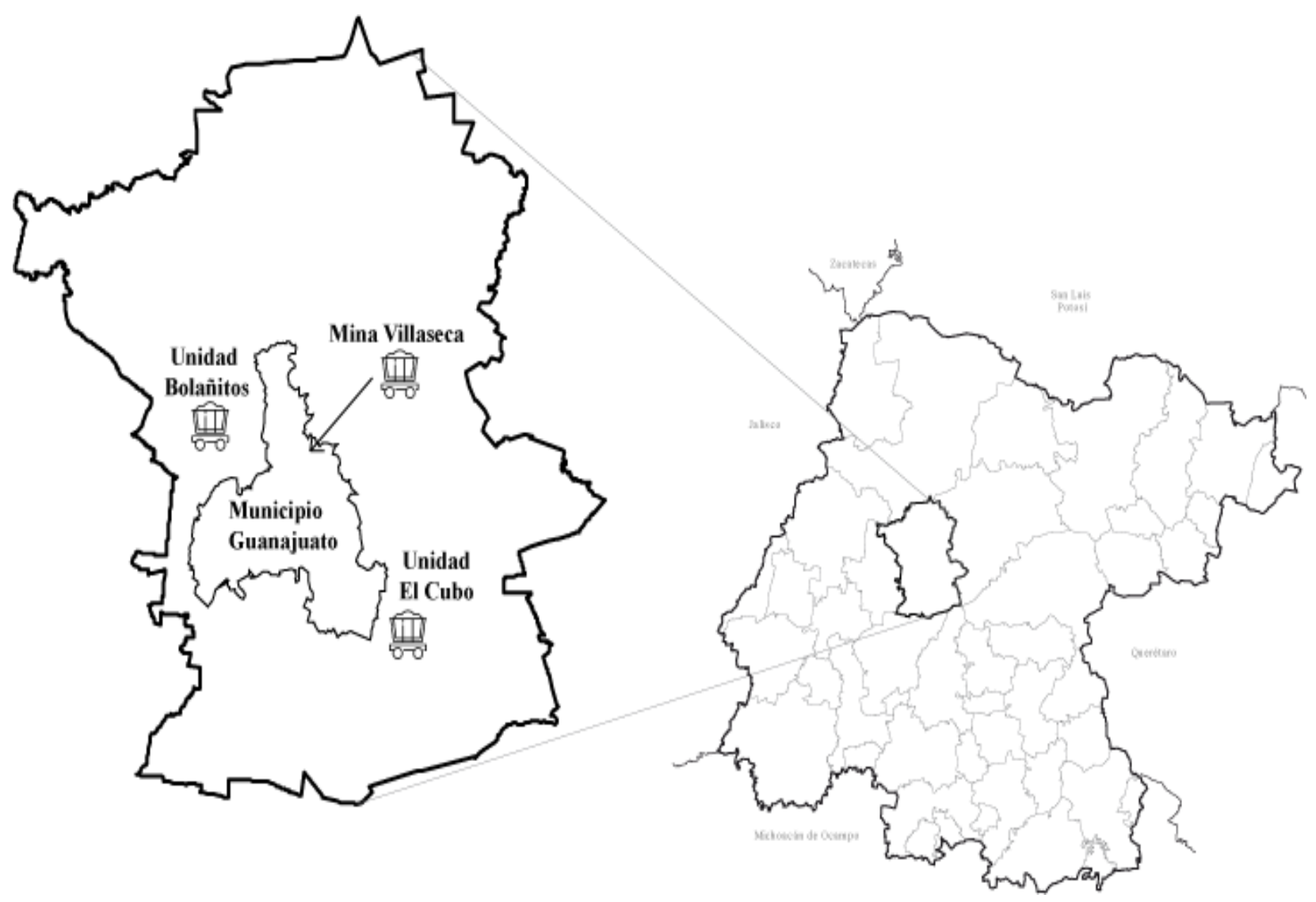

Figura 3. Mapa de ubicación de los proyectos mineros metálicos activos en Guanajuato. Fuente: Elaboración propia con base en datos de SGM, 2017.

El valor del oro y la plata obtenido en estos proyectos durante el año 2017 fue de 3234 millones de pesos, lo que representa $4.4 \%$ del que generó el país ese mismo año (SGM, 2017). Ambas corporaciones se encuentran ubicadas en el municipio de Guanajuato, a 30 minutos de la ciudad (entre 12 y 20 kilómetros de distancia cada mina respecto al centro del municipio).

Por otra parte, la aportación de estas no es representativa del Producto Interno Bruto (PIB) estatal, pues generan menos de $2 \%$ y emplean a $0.01 \%$ de sus habitantes, por lo que tienen mínimos o nulos efectos en la generación de ingresos locales (INEGI, 2019); aunque, la contribución de Guanajuato al PIB minero nacional es significativo (6 \%) (CAMIMEX, 2019). Es decir, este sector se disocia de la economía de la entidad y se centra en fortalecer la industria nacional, la cual se orienta hacia el sector externo.

De forma adicional a esta falta de capacidad para beneficiar a los municipios o a la provincia, las autoridades estatales no han dado seguimiento a las denuncias vecinales sobre los riesgos que

90

Aleida Azamar Alonso

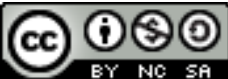

Revista Economía y Sociedad by Universidad Nacional is licensed under a CreativeCommons Reconocimiento-NoComercial- 
ocasionan estos proyectos, ya que se encuentran muy cerca de la ciudad (Espinosa, 2016). Estas características, así como el hecho que solo sean dos compañías las que están activas, son adecuadas para evaluar los elementos que podrían determinar si existe un proceso de extractivismo monopolista en desarrollo dentro de esta región. El análisis se centra únicamente en la empresa Endeavour Silver, pues tiene mayor cantidad de minas que Great Panther Silver LTD, por lo que su influencia regional será mayor.

\section{Endeavour Silver Corp.}

Esta compañía tiene su matriz en Vancouver, Canadá, fue fundada para realizar labores de explotación minera en América Latina, específicamente orientada al aprovechamiento de metales preciosos. Es de reciente creación, se fundó en el 2003 y comenzó a operar en 2007 en el estado de Guanajuato ${ }^{11}$ en la unidad minera ${ }^{12}$ conocida como Bolañitos; asimismo, es dueña de otra unidad en la misma entidad llamada El Cubo y produce desde 2012 (ESC, 2017).

\section{Unidad minera Bolañitos}

Bolañitos comienza operaciones en 1968 por parte del Grupo Peñoles, pero las capacidades y reservas de las diferentes minas en el lugar decrecieron lo que provocó pérdidas para la compañía, e impulsó a la empresa a transferir la concesión, dicha transferencia se realizó en 2007 a Endeavour.

Bolañitos fue la primera unidad que la compañía canadiense obtuvo en Guanajuato. La extensión de la concesión es de 2533 hectáreas y en esta operan tres minas: Bolañitos, Lucero y Asunción; además existen otras dos que están inactivas (Cebada y Golondrinas) (Robles, 2018). En Bolañitos se construyó una presa de jales y también una planta de beneficio (véase Figura 4).

\footnotetext{
${ }^{11}$ Aunque esta empresa ya tenía un proyecto en el estado de Durango que comenzó a funcionar en 2004 y otros proyectos de exploración antes 2007, Actualmente cuenta con doce prospectos, que incluyen los dos tratados en este trabajo.

${ }^{12}$ Una unidad minera se refiere a la agrupación de varias minas por parte de la misma empresa, así como una planta de beneficio.
} 


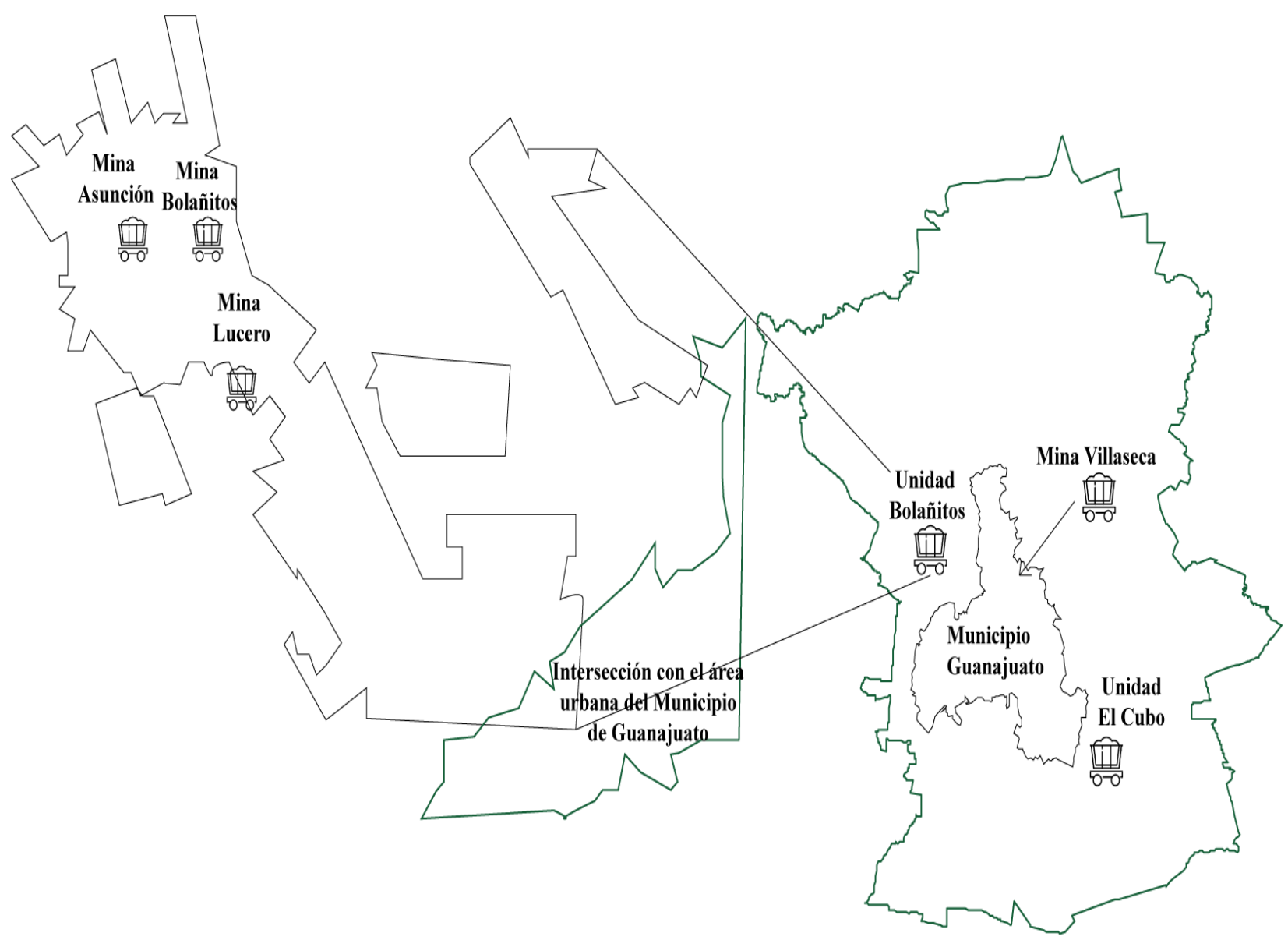

Figura 4. Mapa de la unidad minera Bolañitos y sus minas activas*. Fuente: Elaboración propia con base en datos de Robles, 2018, p. 94. Nota: En color verde el contorno de la entidad y en color negro el contorno de la concesión minera.

En esta unidad se obtiene plata y oro de depósitos subterráneos por lo que se emplean dos técnicas de extracción. La primera es de corte y relleno, en la cual cuatro personas sostienen un taladro que puede perforar de uno a dos metros de profundidad, después deben ser rellenados con piedras para mantener la capacidad de sostén de la mina. Esta forma de trabajo es peligrosa y poco productiva en comparación con el segundo método, pero se sigue usando, pues en algunos espacios es imposible hacerlo de otra manera por el poco campo.

El segundo método es denominado de barrenación larga y para ello se utiliza un pequeño tractor llamado jumbo que es movilizado por una sola persona, el cual puede abarcar hasta 12 metros en cada ocasión. Aunque este método es mucho más barato y productivo que el primero, lo cierto es que genera un mayor nivel de destrucción y desperdicio de material al no enfocarse en una sola área.

92 
A pesar de los riesgos para los trabajadores y para el medio ambiente, el uso de la barrenación larga en conjunto con la práctica de corte y relleno se ha llevado a cabo desde el año 2007 y ha generado un incremento notable en la capacidad operativa.

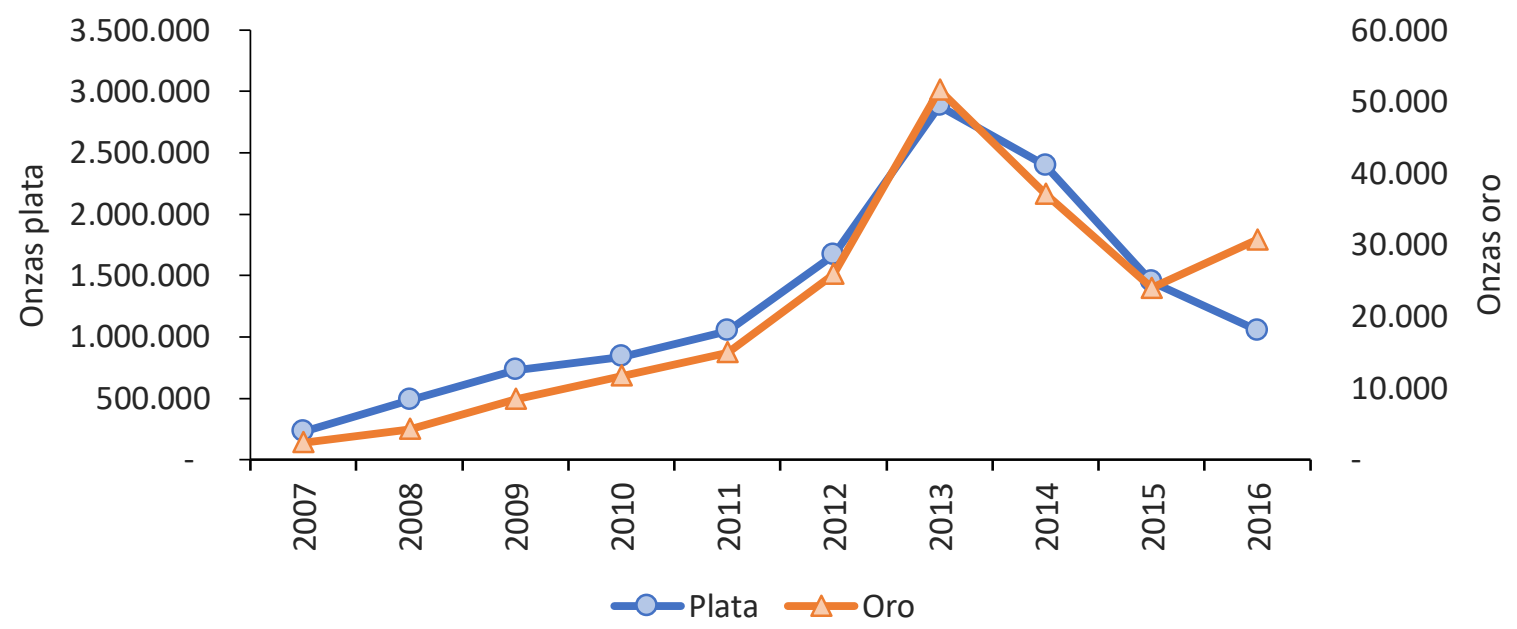

Figura 5. Producción anual de onzas de plata y oro en la unidad Bolañitos, 2007-2016. Fuente: elaboración propia con datos de ESC, 2017.

Como se observa en la Figura 5, hasta el 2013 se mantuvo un crecimiento productivo para ambos metales, los cuales después comenzaron un lento declive (que se ha mantenido hasta la actualidad para la plata, aunque en el caso del oro ha tenido una ligera recuperación en 2016). Este comportamiento responde a situaciones de carácter exterior: la caída en los precios de los minerales y la disminución en la demanda asiática de los mismos. Y es que la totalidad de la producción, sin ningún tratamiento, es exportada inmediatamente después de su purificación hacia Corea del Sur para la creación de productos de alta tecnología (ESC, 2017).

\section{Unidad minera El Cubo}

Esta unidad data de 1970 como negocio familiar mexicano, la cual posteriormente fue vendida a la empresa nacional llamada Mexgold Resources Inc. en 2004 y más tarde se convertiría en una subsidiaria de la compañía canadiense Gammon Gold en 2006 (Robles, 2018).

Después de que la empresa extranjera quedó como dueña de la mina se suscitaron varios problemas con los trabajadores debido a la violación del contrato laboral que se tenía y a la imposición de normas de trabajo diferentes a las acordadas, ya que se exigían 10 horas diarias de labores que supuestamente serían compensadas con las utilidades, cosa que no sucedió y que 
condujo a una huelga en la que la compañía cerró la mina sin justificación alguna y, finalmente, se vendió sin indemnizar a los trabajadores. Dicho acto no fue sancionado por el Estado.

En 2012 la empresa es adquirida por Endeavour para recomenzar el proyecto de explotación minero, ya que de acuerdo con sus registros existen varias vetas que no han sido exploradas y aprovechadas del todo, por lo que esta puede ser mucho más productiva de lo que ha sido hasta ahora.

Esta unidad es la más cercana al municipio de Guanajuato, ya que solamente le separan 10 kilómetros de la ciudad. Además, tiene tres minas activas en un área de 8141 ha que conforman la concesión (SGM, 2017). Estas son: Dolores, San Cecilia y San Nicolás; asimismo, igual que en el caso anterior también cuenta con una presa de jales (véase Figura 6).

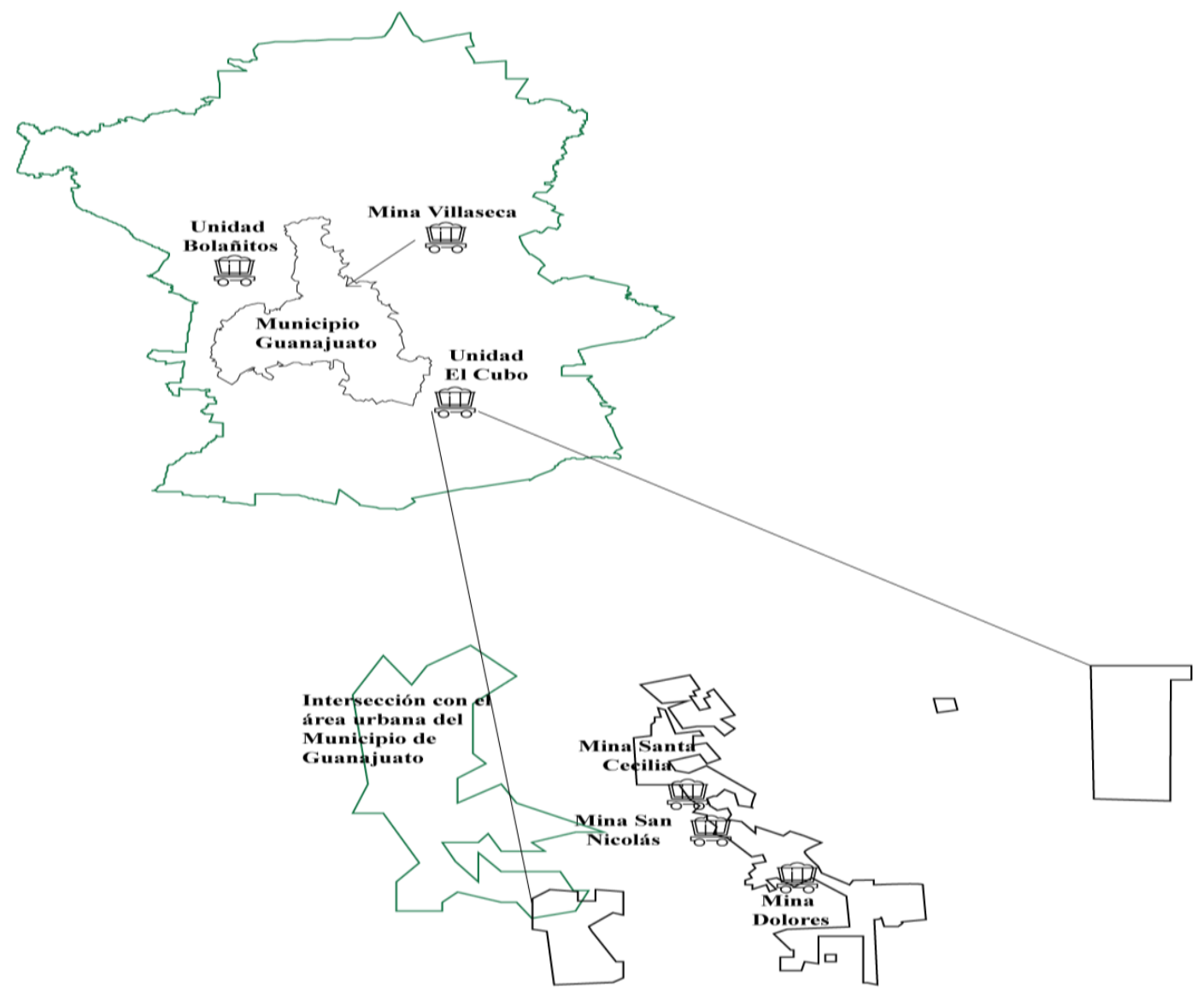

Figura 6. Mapa de la unidad minera El Cubo y sus minas activas*. Fuente: elaboración propia con base en datos de Robles, 2018, p. 108. Nota: en color verde el contorno de la entidad y en color negro el contorno de la concesión minera.

94 
Dicha unidad también tiene como principales recursos el oro y la plata, la única diferencia es que debido a las inclinaciones y formas geográficas de las minas estas utilizan principalmente la técnica de barrenación larga. Este mayor nivel de uso del tractor se refleja en el incremento de la producción, pues en contraste con Bolañitos la extracción de recursos ha disminuido solamente entre 2015 y 2016, respondiendo esencialmente al uso intensivo del jumbo.

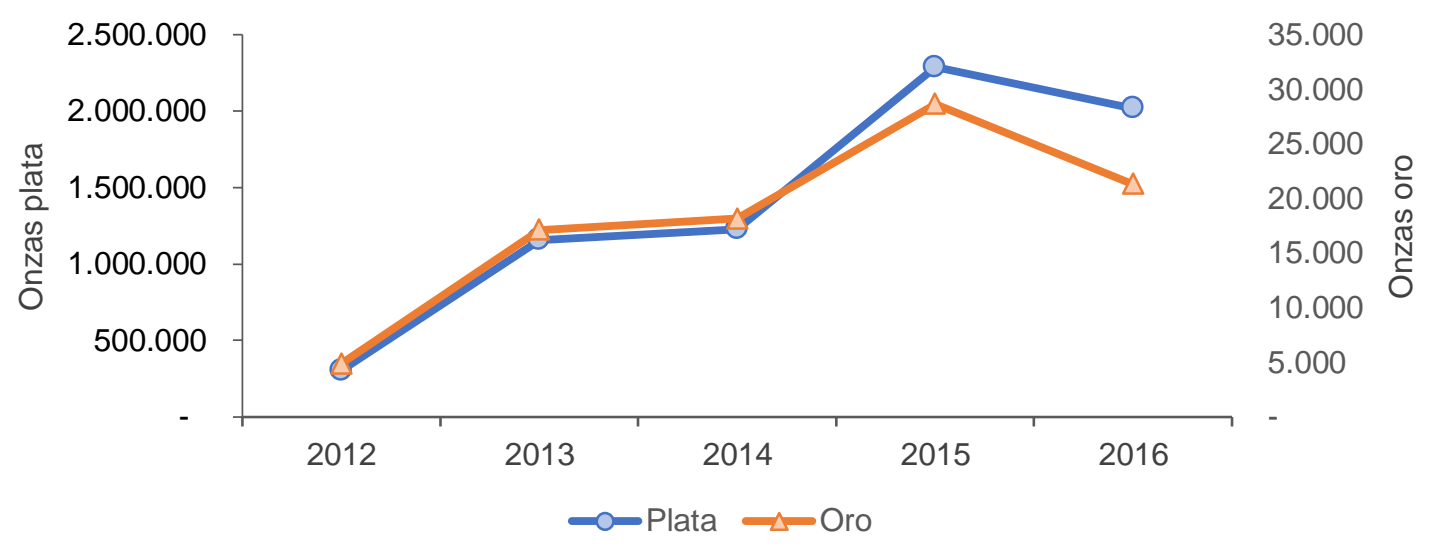

Figura 7. Producción anual de onzas de plata y oro en la unidad el Cubo, 20072016. Fuente: elaboración con base en datos de ESC, 2017.

Como se observa en la Figura 7, esta unidad ha resultado ser mucho más productiva con las onzas de plata que Bolañitos, pero en relación con la producción de oro está por debajo aún en sus máximos niveles que presentó en el 2015. A pesar de lo anterior, la duplicación en la productividad de plata le da un mayor valor estratégico y económico, ya que exporta la totalidad de lo que se obtiene a China y Corea del Sur para la elaboración de baterías y materiales médicos de alta tecnología (ESC, 2017).

\section{Reflexiones finales}

El corporativismo es la respuesta social ante la monopolización del poder (económico, político, bélico, etc.) que tiene el Estado, por lo cual el surgimiento de organizaciones corporativistas responde a la carencia de autonomía de los individuos o de los mercados ante la administración pública, para incrementar el margen de acción de sus derechos.

Aunque, en la actualidad, este tipo de formación en entornos empresariales, como en el caso de la minería en el estado de Guanajuato, tiende a fortalecerse a través de la acumulación territorial y de beneficios económicos. La institucionalización de estas redes empresariales corporativistas se divorcia del bienestar social y se concentra en nutrir al mercado mundial, lo que se observa en Guanajuato al señalar que menos del $1 \%$ de sus habitantes están empleados por el sector minero 
extractivo, además de que el impacto económico es nulo para las finanzas de la entidad, pero paradójicamente impulsa el PIB del sector minero nacional, el cual está orientado al comercio internacional.

El extractivismo minero monopolista en Guanajuato es un proceso innegable. Las concesiones en este territorio ocupan $15 \%$ de la superficie total y pertenecen a doce empresas, diez de las cuales son canadienses, pero las compañías activas en proceso de extracción son solamente dos.

Lo anterior se presenta en el marco de un incremento notable de las concesiones para la explotación de estos recursos en el municipio de Guanajuato ( $81 \%$ en doce años); lo cual es responsabilidad e interés del Estado en todos sus niveles, pues la autoridad federal aprueba dichos permisos y los encargados locales ratifican el apoyo al permitir la operación de estos proyectos.

Para el caso particular de las unidades mineras Bolañitos y El Cubo, ambas son de la compañía canadiense: ESC, la cual acapara dos terceras partes de la producción total de minerales metálicos en la entidad, por lo que su posición dominante dificultaría el desarrollo de un universo empresarial diversificado como lo propone la teoría clásica de la economía. El otro participante del sector del Estado para este tipo de recursos también es foráneo, ambas empresas pertenecen a Canadá, país que además domina de forma contundente este tipo de aprovechamiento, ya que más del $60 \%$ de los proyectos activos actualmente en México pertenecen a esa nación.

Es notorio que no se está contribuyendo, positivamente, a la economía de la entidad, a través de estos proyectos, pero sí se está impulsando la macroeconomía del país, por lo que, en este caso, Guanajuato actúa como un espacio periférico que solamente será considerado en el marco de explotación y despojo territorial para beneficio del Gobierno central. Es así como se establece, primero, el apoyo absoluto del poder público a las empresas extractivas para llevar a cabo sus actividades, aunque esto no ayude a los actores locales, y segundo, la aprobación tácita de estructuras corporativistas monopolistas carentes de contrapesos que pueden influir en el desarrollo de la política nacional.

96 


\section{Referencias}

Acosta, A. (2009). La maldición de la abundancia. (Primera edición). Quito, Ecuador: Ediciones Abya-Yala. Recuperado de http://www.rebelion.org/docs/122604.pdf

Acosta, A. (2012). Extractivismo y neoextractivismo: Dos caras de la misma maldición. En Lang, M. \& Mokrani, D. (Primera edición, 83-118.). Más allá del desarrollo. Quito, Ecuador: Ediciones ABYA YALA, Fundación Rosa Luxemburgo. Recuperado de http://www.rosalux.org.mx/docs/Mas_alla_del_desarrollo.pdf

Alimonda, H. (2011). La colonialidad de la naturaleza. Una aproximación a la Ecología Política Latinoamericana. En Alimonda, H. La naturaleza colonizada: Ecología política y minería en América Latina. (Primera edición, 21-58). Buenos Aires, Argentina: Consejo Latinoamericano de Ciencias Sociales CLACSO, Ediciones CICCUS. Recuperado de: http://biblioteca.clacso.edu.ar/clacso/gt/20120319035504/natura.pdf

Azamar Alonso, A. (2017). Megaminería en México: explotación laboral y acumulación de ganancia. (Primera edición). México: Editorial ITACA-Universidad Autónoma Metropolitana. Recuperado

de https://books.google.co.cr/books/about/Megaminer\%C3\%ADa en M\%C3\%A9xico.h tml id $=3$ PwuQEACAAJ \& redir esc $=y$

Baran, P. (1987). La economía política del crecimiento. (Quinta edición). México: Fondo de Cultura Económica.

Baran, P. \& Sweezy, P. (2006). El capital monopolista: ensayo sobre el orden económico y social de Estados Unidos. (Vigésima primera edición). México: Siglo XXI editores. Recuperado de https://books.google.com.mx/books/about/El_capital_monopolista.html?id=ONDoq

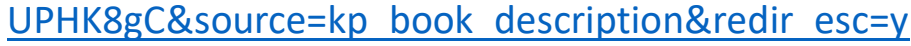

Bellamy Foster, J. (2014). The theory of monopoly capitalism: An Elaboration of Marxian Political Economy. (Primera edición). Nueva York: Monthly Review Press. Recuperado de: https://monthlyreview.org/product/theory of monopoly capitalism/ 
Borgtoft Pedersen, G. \& Baslev, H. (1993). Palmas útiles. Especies ecuatorianas para agroforestería y extractivismo. (Primera edición). Quito, Ecuador: Ediciones ABYAYALA.

Recuperado

de: http://biblioteca.udla.edu.ec/client/en_US/default/search/detailnonmodal/ent:\$00 2f\$002fSD ILS\$002f0\$002fSD ILS:22358/ada?qu=Borgtoft+Pedersen\%2C+Henrik\&ic =true $\&$ te $=\mid \mathrm{LS} \& \mathrm{ps}=300$

Brunhes, J. (1920). Human Geography: An attempt at a positive classification principles and examples. (Primera edición). Chicago: Rand Mc Nally \& Company. Recuperado de https://babel.hathitrust.org/cgi/pt?id=yale.39002004957982;view=1up;seq=9

Cámara Minera de México. (2019). Mapa de Indicadores Mineros: Base de datos en línea. Disponible en: https://camimex.org.mx/index.php/mapa nacional/

Ceceña Martorella, A. E. (2016). La territorialidad de las corporaciones. En Ceceña Martorella, A. E. y Ornelas Bernal, R. Las corporaciones y la economía-mundo: El capitalismo monopolista y la economía mexicana en retrospectiva. (Primera edición, 108-133). México: Instituto de Investigaciones Económicas, Siglo XXI editores. Recuperado de: http://www.libros.unam.mx/las-corporaciones-y-la-economia-mundo-elcapitalismo-monopolista-y-la-economia-mexicana-9786070280771-libro.html

Ceceña Martorella, A. E. \& Barreda, A. (1995). Producción estratégica y hegemonía mundial. México: Siglo XXI editores. Recuperado de: https://books.google.com.ec/books?id=rFbr7FYKEOAC\&printsec=frontcover\&hl=es\# $\mathrm{v}=$ onepage $\& \mathrm{q} \& \mathrm{f}=\mathrm{false}$

Ceceña Martorella, A. E. \& Ornelas Bernal, R. (2016). Las corporaciones y la economía-mundo: El capitalismo monopolista y la economía mexicana en retrospectiva. (Primera edición). México: Instituto de Investigaciones Económicas, Siglo XXI Editores. Recuperado de: http://www.libros.unam.mx/las-corporaciones-y-la-economia-mundo-elcapitalismo-monopolista-y-la-economia-mexicana-9786070280771-libro.html

De la Garza, E. (2006). Corporativismo sindical y modelo neoliberal en México. En G. Otero, México en transición: globalismo neoliberal, Estado y sociedad civil, Colección América Latina y el Nuevo Orden Mundial. (Primera edición, 177-193). México: Miguel Ángel Porrúa, UAZ.

Dufour, D. L. (1990). Use of Tropical Rainforests by Native Amazonians: These sophisticated and complex agricultural systems can serve as models of sustainable agroecosystems. BioScience, 9(40), 652-659. doi: https://doi.org/10.2307/1311432

98

Aleida Azamar Alonso

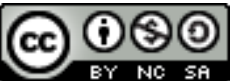

Revista Economía y Sociedad by Universidad Nacional is licensed under a CreativeCommons Reconocimiento-NoComercialCompartirlgual 4.0 Internacional License. Creado a partir de la obra en http://www.revistas.una.ac.cr/index.php/economia 
Endeavour Silver Corporation. (2017). Informe anual de discusión gerencial y análisis. Recuperado de: https://www.edrsilver.com/Spanish/informacion-para-inversionistas/reportesfinancieros/default.aspx

Espinosa, V. (2016). Ordenan al INAH y PROFEPA revisar riesgos por actividad minera en comunidad guanajuatense. PROCESO. Recuperado de: https://www.proceso.com.mx/466752/ordenan-al-inah-profepa-revisar-riesgosactividad-minera-en-comunidad-guanajuatense

Fearnside, P. M. (1989). Extractive Reserves in Bazilian Amazonia: An opportunity to maintain tropical rain forest under sustainable use. BioScience, 6(39), 387-393. Recuperado de: https://doi.org/10.2307/1311068

Gudynas, E. (2014). Conflictos y extractivismos: conceptos, contenidos y dinámicas. Decursos, Revista en Ciencias Sociales, 27-28, 79-115. Recuperado de: http://gudynas.com/wpcontent/uploads/GudynasConflictosExtractrivismosConceptosDecs14.pdf

Instituto Nacional de Estadística y Geografía. (2019). Base de datos en línea. Recuperada de: http://www.inegi.org. $\mathrm{mx} /$

Lijphart, A. (2000). Modelos de democracia, formas de gobierno y resultados en 36 países. (Primera edición). Barcelona, España: Editorial Ariel.

Marx, C. (2003). El capital: crítica de la economía política. Tomo 1/Volumen 2. El proceso de producción del capital. (Vigésima segunda Edición). México: Siglo XXI editores. Recuperado de: https://books.google.com.mx/books?id=H9AjKhwnC9IC\&printsec=frontcover\&sourc e=gbs ge summary $r \& c a d=0 \# v=$ onepage $\& q \& f=$ false

Marx, C. y Engels, F. (1980). Materiales para la historia de América Latina. (Cuarta edición). Argentina: Ediciones Pasado y Presente. Recuperado de: https://books.google.com.mx/books?id=bw3qQQAACAAJ\&dq=\%22Materiales+para +la+historia+de+Am\%C3\%A9rica+Latina\%22\&hl=es-419\&sa=X\&ved=0ahUKEwiK8yh0aDhAhXr1IMKHd0dCn4Q6AEIKTAA

Materialflows. (2019). Base de datos en línea. Recuperado de: http://www.materialflows.net/

Nadal, A. (2015). Capital natural: una metáfora peligrosa. Recuperado de https://www.jornada.com.mx/2015/11/11/opinion/036a1eco 
Rivera, G. (2017). 8 empresas que dominan la minería mexicana. Recuperado de: http://www.manufactura.mx/industria/2017/07/11/extranjeros-dominan-lamineria-mexicana-y-el-empleo

Robles Rodríguez, K. A. (2018). Impacto territorial socio-económico derivado de la presencia de las empresas Endeavour Silver Corporation y Great Panther Silver Limited en la minería del Municipio de Guanajuato, Guanajuato, 1980-2015. (Tesis que para obtener el título de Licenciado en Geografía). México: Universidad Nacional Autónoma de México.

Romeo LopCam (2018). Mapa interactivo de concesiones mineras en México. Recuperado de: https://romeolopcam.carto.com/

Ruiz Pérez, M., Sayer, J. A., \& Cohen Jehoram, S. (1993). El extractivismo en América Latina. Conclusiones y recomendaciones del Taller UICN-CCE. Unión Internacional para la Conservación de la Naturaleza. Recuperado de: https://books.google.com.mx/books?id=MdOaiggeiggC\&dq=Ruiz+P\%C3\%A9rez,+M., + Sayer, + +J.+A., $+26+$ Cohen+Jehoram, + S.+(1993).+El+extractivismo+en+Am\%C3\%A9r ica+Latina.+Conclusiones+y+recomendaciones+del+Taller+UICN-

CCE\&source $=$ gbs navlinks $S$

Schmitter, P. (1974). Still the Century of Corporatism? The Review of Politics, 1(36), 85-131. doi: https://doi.org/10.1017/S0034670500022178

Servicio Geológico Mexicano. (2017). Panorama minero del Estado de Guanajuato. Recuperado de: http://www.sgm.gob.mx/pdfs/GUANAJUATO.pdf

Slipak, A. M. (2013). ¿De qué hablamos cuando hablamos de reprimarización?: Un aporte al debate sobre la discusión del modelo de desarrollo. Efectuado en VI Jornadas de Economía Crítica, Universidad Nacional de Cuyo, Buenos Aires, Argentina. Recuperado de: https://www.academia.edu/7188437/_De_qu\%C3\%A9 hablamos cuando hablamo s_de reprimarizaci\%C3\%B3n_Un_aporte_al debate_sobre_la_discusi\%C3\%B3n del modelo de desarrollo

Vladimir Lenin, I. (2012). Imperialismo: la fase superior del capitalismo. (Primera edición). España: Editorial Penguin Random House. Recuperado de: https://books.google.com.mx/books?id=Q5XEFD2GjR4C\&dq=\%22Imperialismo\%3A \%20la\%20fase\%20superior\%20del\%20capitalismo\%22\&source=gbs book other ver sions

100 